\title{
The Assessment of the Efficacy of Carboxymethyl Chitosan in the Surgical Treatment of Carpal Tunnel Syndrome: A Retrospective Study
}

\author{
Timur Yildirım ${ }^{1}$ \\ ${ }^{1}$ Department of Neurosurgery KTO Karatay University, Medical School, Konya , Turkey \\ Received: 14 March 2020, Accepted: 12 April 2020, Published online: 30 April 2020 \\ (C) Ordu University Institute of Health Sciences, Turkey, 2020
}

\begin{abstract}
Objective: Carpal Tunnel Syndrome (CTS) is the most commonly encountered entrapment neuropathy of the upper extremity characterized by the compression of the median nerve at the level of the wrist. The objective of the treatment is to eliminate the symptoms stemming from compression and to preserve the neurological function by relieving the median nerve of pressure. Conservative and surgical treatment methods are used effectively in Carpal Tunnel Syndrome. Anti- Adhesion barriers are used during surgery. The aim of this study is to investigate the postoperative effectiveness of carboxymethyl chitosan and methylprednisolone used intraoperatively.

Methods: 87 patient files (26 males, 61 female) who were operated with the diagnosis of moderate degree Carpal Tunnel Syndrome. Peroperative local carboxymethyl chitosan gel was applied following the surgical decompression of the transverse carpal ligament in 32 patients, after surgical decompression peroperative local methylprednisolone was applied in 28 patients and only surgical decompression was performed in 27 patients. Preop and post op complaints of patients were evaluated using Symptom Severity Scale (SSS), Functional Status Score (FSS), Boston Symptom Severity Scale (BSSS), Visual Analogue Scale (VAS).

Results: When pre and postoperative CNS values were evaluated according to gender and intervention groups, male patients had higher CNS values than female patients $(\mathrm{P}=0.046)$. When preop and postop FSS values were evaluated according to gender and intervention groups, FSS values decreased significantly in all surgical intervention groups compared to preoperative values $(\mathrm{P}<0.001)$. When preop and postop VAS scores were assessed with emphasis on sex and intervention groups, VAS scores of the patients in all intervention groups showed a significant decrease as compared to preoperative scores in a time dependent manner $(\mathrm{P}<0,001)$.

Conclusion: We compared the groups treated with CMCS and methyl prednisolone during surgical interventions for carpal tunnel syndrome with those who had undergone only surgery; we see that there was a significant decrease in the symptoms at three months.
\end{abstract}

Key words: Carpal Tunnel Syndrome, Carboxymethyl Chitosan, Surgical Treatment

Suggested Citation: Yildirim T. The Assessment of the Efficacy of Carboxymethyl Chitosan in the Surgical Treatment of Carpal Tunnel Syndrome: a Retrospective Study Middle Black Sea Journal of Health Science, 2020; 6(1):76-82.

Address for correspondence/reprints:

Timur Yıldırım

E-mail: mdtimur@hotmail.com

Telephone number: +90 (505) 3592385

DOI: $10.19127 / \mathrm{mbsjohs} .703740$

ORCID-ID 0000-0002-2334-2711 


\section{Introduction}

Carpal Tunnel Syndrome (CTS) is the most commonly encountered entrapment neuropathy of the upper extremity characterized by the compression of the median nerve at the level of the wrist (Hadler, 1993). Its main symptoms are numbness of the first three fingers that specifically appear during nighttime, and findings due to pain in the forearm and loss of strength (Gerritsen et al., 2002). The objective of the treatment is to eliminate the symptoms stemming from compression and to preserve the neurological function by relieving the median nerve of pressure. Conservative and surgical treatment methods are used effectively to this end. Surgery is indicated when conservative treatment methods become insufficient or if there is motor weakness and thenar atrophy (Hadler, 1993; Scholten et al., 1997). Adhesion preventing barriers used during surgery are also effective after surgery by helping people experience less pain due to adhesions. The aim of this study is to investigate the postoperative efficacy of carboxymethyl chitosan and methylprednisolone used intraoperatively for adhesion prevention in patients diagnosed with CTS clinically and electrophysiologically and treated with surgery.

\section{Methods}

In this study, after obtaining the relevant clinical ethical committee approval, we retrospectively analyzed 87 patient files ( 26 male, 61 female) who were operated with the diagnosis of moderate degree Carpal Tunnel Syndrome (maximum duration of complaints: one year, absence of thenar muscle atrophy and weakness, absence of denervation in electromyography, mild effects on nerve conduction.). Peroperative local carboxymethyl chitosan gel was applied following the surgical decompression of the transverse carpal ligament in 32 patients, after surgical decompression peroperative local methylprednisolone was applied in 28 patients and only surgical decompression was performed in 27 patients.

In this study, exclusion criteria were the evidence of pronounced abductor pollicis weakness or significant thenar wasting, prior carpal tunnel surgery on the affected side, the use of narcotic analgesics, a history of wrist or hand fracture on the symptomatic limb, having a current pregnancy or being less than 3 months postpartum, corticosteroid injection into the carpal tunnel within 3 months and having severe or mild carpal tunnel syndrome.

For questioning the symptoms of the patients, 11 question Symptom Severity Scale (SSS) and 8 question Functional Status Score (FSS) were used to evaluate Boston Symptom Severity Scale (BSSS) that has a maximum score of 5 , together with Visual Analog Scale (VAS) (6). Visual Analog Scale (VAS) was used to determine the severity of pain (5). VAS is usually a line of $10 \mathrm{~cm}$, either horizontal or vertical, from "No Pain" to "Unbearable Pain". According to this scale, 0: no pain, 1-3: mild pain, 4-6: moderate pain, 7-10 severe pain. The pain scores of patients before surgery and 3 months after surgery were recorded for statistical evaluation.

\section{Statistical analysis}

All statistical analyses were performed with SPSS 20.0 Version statistical package program (SPSS Inc., Chicago, IL, USA). Firstly, the data (ages and the scores of Symptom Severity Scale, Functional Status Score and VAS) were analyzed using Levene's test and the Shapiro-Wilk test for equality of variance and normality assumption, respectively $(\mathrm{P}>0.05)$. Secondly, two-way ANOVA was applied to determine whether there were differences among ages for the two groups (Applications: Surgery+CC, surgery+methylprednisolone and surgery group; gender: male and female). Also, data (Symptom severity scale, Functional Status Score and VAS) obtained from two different time periods (preoperative and postoperative) were analyzed for the effects of the intervention methods and gender using repeated measurements of ANOVA and the means were compared by Tukey test. If the $p$ value was below 0.05 , the results were considered statistically significant. The research findings were expressed as n, mean and standard deviation.

\section{Results}

When preoperative and postoperative SSS values were evaluated based on sex and intervention groups; male patients were found to have higher SSS values than female patients $(\mathrm{P}=0,046)$. As time passed, preoperative SSS values of the patients decreased significantly in all of the surgical intervention groups $(\mathrm{P}<0,001)$. However, the groups that had undergone Surgery+CC and surgery+methylprednisolone had higher decreases in perceived SSS values as compared to patients undergoing surgical intervention only $(\mathrm{P}=0,002$; Table 1$)$.

Based on sex and intervention groups, when preop and postop FSS values were assessed, FSS values have significantly decreased compared with the preoperative values in all the surgical intervention groups $(\mathrm{P}<0,001)$. However, the decrease perceived in the FSS values of patients having Surgery $+\mathrm{CC}$ and surgery+methylprednisolone was more significant than those perceived by patients who had undergone 
surgery only $(\mathrm{P}=0,003)$. Perceived FSS values before and after surgery did not differ between male and female patients ( $\mathrm{P}=0,590$; Table 2).

When preop and postop VAS scores were assessed with emphasis on sex and intervention groups, VAS scores of the patients in all intervention groups showed a significant decrease as compared to preoperative scores in a time dependent manner $(\mathrm{P}<0,001)$. There was no difference between male and female patients in perceived VAS scores before and after surgery ( $\mathrm{P}=0,806$; Table 3$)$.

Table 1. preoperative and postoperative SSS values

\begin{tabular}{|c|c|c|c|c|c|c|c|}
\hline \multirow{3}{*}{ Applications (A) } & \multirow{3}{*}{ Gender (G) } & \multicolumn{6}{|c|}{ Time $(\mathrm{T})$} \\
\hline & & \multicolumn{3}{|c|}{ PREOP } & \multicolumn{3}{|c|}{ POSTOP3.AY } \\
\hline & & $\mathrm{n}$ & Mean & SD* & $\mathrm{n}$ & Mean & SD \\
\hline \multicolumn{8}{|l|}{ SSS } \\
\hline \multirow[t]{3}{*}{ Surgery+CMCS } & Female & 24 & 41.17 & 4.53 & 24 & 17.54 & 3.46 \\
\hline & Male & 8 & 41.00 & 4.54 & 8 & 17.38 & 1.92 \\
\hline & Total & 32 & 41.13 & 4.46 & 32 & 17.50 & 3.12 \\
\hline \multirow[t]{3}{*}{ Surgery+MP } & Female & 18 & 39.78 & 3.93 & 18 & 18.89 & 2.91 \\
\hline & Male & 10 & 41.80 & 4.05 & 10 & 18.90 & 3.57 \\
\hline & Total & 28 & 40.50 & 4.02 & 28 & 18.89 & 3.10 \\
\hline \multirow[t]{3}{*}{ Surgery } & Female & 19 & 37.79 & 4.63 & 19 & 20.74 & 4.11 \\
\hline & Male & 8 & 40.75 & 4.53 & 8 & 23.38 & 4.69 \\
\hline & Total & 27 & 38.67 & 4.71 & 27 & 21.52 & 4.37 \\
\hline \multirow[t]{3}{*}{ Total } & Female & 61 & 39.70 & 4.55 & 61 & 18.93 & 3.72 \\
\hline & Male & 26 & 41.23 & 4.20 & 26 & 19.81 & 4.25 \\
\hline & Total & 87 & 40.16 & 4.48 & 87 & 19.20 & 3.88 \\
\hline \multicolumn{8}{|l|}{ Variation sources } \\
\hline Effects & $\mathrm{A}$ & $\mathrm{G}$ & $\mathrm{T}$ & $\mathrm{AxG}$ & AxT & GxT & AxGxT \\
\hline P-values & 0.181 & 0.046 & $<0.001$ & 0.144 & 0.002 & 0.591 & 0.824 \\
\hline
\end{tabular}

\section{Discussion}

Carpal tunnel syndrome (CTS) is a spectrum of symptoms resulting from the compression of the median nerve in the carpal tunnel while being the most commonly observed entrapment neuropathy. Initial signs can be only nocturnal pain and paresthesia, as it progresses atrophy of thenar muscles and loss of strength can be added to the clinical presentation (Scholten et al., 1997). As connective tissue is exposed to repetitive stress; ensuing edema, vascular sclerosis and fibrosis are thought to exert mechanical pressure on the median nerve. The compression of the median nerve leads to ischemia and focal demyelization. There is axonal loss in severe cases and "Wallerian degeneration" ensues. Several conservative and surgical methods are used for the treatment of CTS. Conservative treatment for CTS includes rest splints, physical therapy modalities, NSAID, diuretics, local and systemic steroids, pyridoxine and yoga techniques (Yagci et al., 2006, Carlson et al.; 2010).

There are several studies investigating the efficacy of local corticosteroid injections among conservative methods. In a prospective study by Gelbermen et al. 50 hands of 41 patients were evaluated; they used a single injection of steroids together with three weeks of rest splint application obtaining favorable results in CTS patients having mild to moderate symptoms If the complaints of the patient do not improve despite conservative treatment and if muscular atrophy starts then surgical treatment would be required. (Gelbermen et al., 1980). 
Table2. Preoperative and postoperative FDS values

\begin{tabular}{|c|c|c|c|c|c|c|c|}
\hline \multirow{3}{*}{ Applications (A) } & \multirow{3}{*}{ Gender $(\mathrm{G})$} & \multicolumn{6}{|c|}{ Time $(\mathrm{T})$} \\
\hline & & \multicolumn{3}{|c|}{ PREOP } & \multicolumn{3}{|c|}{ POSTOP 3.AY } \\
\hline & & $\mathrm{n}$ & Mean & $\mathrm{SD}^{*}$ & $\mathrm{n}$ & Mean & SD \\
\hline \multicolumn{8}{|l|}{ FDS } \\
\hline \multirow[t]{3}{*}{ Surgery+Cmcs } & Female & 24 & 30.58 & 3.28 & 24 & 13.58 & 3.36 \\
\hline & Male & 8 & 31.00 & 2.83 & 8 & 14.88 & 2.59 \\
\hline & Total & 32 & 30.69 & 3.14 & 32 & 13.91 & 3.20 \\
\hline \multirow[t]{3}{*}{ Surgery+mp } & Female & 18 & 28.39 & 2.77 & 18 & 12.72 & 3.18 \\
\hline & Male & 10 & 29.50 & 2.76 & 10 & 13.20 & 2.70 \\
\hline & Total & 28 & 28.79 & 2.77 & 28 & 12.89 & 2.97 \\
\hline \multirow[t]{6}{*}{ Surgery } & Female & 19 & 26.68 & 4.55 & 19 & 15.53 & 3.22 \\
\hline & Male & 8 & 26.63 & 10.82 & 8 & 14.50 & 2.33 \\
\hline & Total & 27 & 26.67 & 6.77 & 27 & 15.22 & 2.98 \\
\hline & Female & 61 & 28.72 & 3.90 & 61 & 13.93 & 3.41 \\
\hline & Male & 26 & 29.08 & 6.40 & 26 & 14.12 & 2.57 \\
\hline & Total & 87 & 28.83 & 4.75 & 87 & 13.99 & 3.16 \\
\hline \multicolumn{8}{|l|}{ Variation sources } \\
\hline Effects & $A$ & $G$ & $T$ & $A x G$ & $A x T$ & $G x T$ & $A x G x T$ \\
\hline$P$-values & 0.088 & 0.590 & $<0.001$ & 0.650 & 0.003 & 0.845 & 0.812 \\
\hline
\end{tabular}

Table 3. Preoperative and postoperative VAS values

\begin{tabular}{|c|c|c|c|c|c|c|c|}
\hline \multirow{3}{*}{ Applications (A) } & \multirow{3}{*}{ Gender (G) } & \multicolumn{6}{|c|}{ Time $(\mathrm{T})$} \\
\hline & & \multicolumn{3}{|c|}{ PREOP } & \multicolumn{3}{|c|}{ POSTOP3.AY } \\
\hline & & $\mathrm{n}$ & Mean & SD* & $\mathrm{n}$ & Mean & $\mathrm{SD}$ \\
\hline \multicolumn{8}{|l|}{ VAS } \\
\hline \multirow[t]{3}{*}{ Surgery $+\mathrm{cmcs}$} & Female & 24 & 8.83 & 1.09 & 24 & 2.25 & 0.85 \\
\hline & Male & 8 & 8.88 & 1.13 & 8 & 2.38 & 0.52 \\
\hline & Total & 32 & 8.84 & 1.08 & 32 & 2.28 & 0.77 \\
\hline \multirow[t]{3}{*}{ Surgery+mp } & Female & 18 & 8.94 & 1.06 & 18 & 2.61 & 0.98 \\
\hline & Male & 10 & 8.80 & 0.92 & 10 & 1.90 & 0.88 \\
\hline & Total & 28 & 8.89 & 0.99 & 28 & 2.36 & 0.99 \\
\hline \multirow[t]{3}{*}{ Surgery } & Female & 19 & 8.58 & 0.84 & 19 & 2.95 & 0.62 \\
\hline & Male & 8 & 9.00 & 0.76 & 8 & 3.00 & 0.93 \\
\hline & Total & 27 & 8.70 & 0.82 & 27 & 2.96 & 0.71 \\
\hline Total & Female & 61 & 8.79 & 1.00 & 61 & 2.57 & 0.87 \\
\hline
\end{tabular}


At later stages of the disease, atrophy might develop in the nerve fibers distal to the compression due to fibrosis taking place in the epineural and perineural components of the nerve tissue thereby resulting in unfavorable outcomes if the surgical intervention is to be delayed. Furthermore, the symptoms might not resolve completely due to adhesions developing after surgery (Phalen 1970; Ulvi et al., 2004).

Adhesions developing after surgery are independent of hemorrhage; fibrinogen that is activated by tissue factors (tissue thromboplastinFactor III) released from the injured tissue form a fibrin gel matrix initiating the process. Fibrin polymers are initially soluble, if they stay in the tissue for a long while they get in contact with certain coagulation factors becoming insoluble and they form the fibrin gel matrix (Rodgers and Dizerega ,1993; Holmdahl et al., 1996). This fluid called serous exudate is required for wound healing and by coagulating within the first 3 hours it results in the formation of adhesions between the neighboring organs. If fibrinolytic activity is insufficient and if the formed adhesion stays for longer than 3 days, fibroblastic proliferation appears and these convert into permanent adhesions. Within the first 10 days following the surgery, the order of processes is such that fibrin matrix is replaced by granulation tissue, fibroblasts and collagen accumulate and they get organized within the adhesions. 1-2 months after the injury adhesion formation mechanisms are complete with the organization of collagen fibrils (Milligan and Raftery, 1974; Raftery 1981).

Mechanical, thermal and electrical applications used during surgical procedures cause damage in anatomical layers resulting in the initiation of inflammation. This decreases fibrinolytic activity and expedites the formation of adhesion. Development of the surgical technique, utilization of adhesion preventing drugs and separation of tissues from each other during the healing process can decrease the adhesions (Schnuriger et al., 2011). Ideal adhesion barriers should not hinder wound healing, should be non-reactive, should be effective in the presence of bodily fluids and blood, should be easy to use and bioabsorbable. In the location it is placed, it should be able to separate those tissues that can form adhesions throughout the first seven days when the wound healing is most intensive (Burns et al., 1996; Koc et al., 2003).

Steroids are reported to prevent the formation of adhesions by impeding the accumulation of fibrin and collagen (Alkan et al., 2007). They accomplish this by inhibiting the first inflammatory response to tissue damage; by preventing vascular permeability and fibroblast proliferation (Alkan et al., 2007). Dexamethasone is a steroidal anti-inflammatory agent that is in combination with sodium carboxymethylcellulose; it can prevent the formation of adhesions, impede the migration of inflammatory cells and later it has been shown to decrease the proliferation of fibroblasts (Durmus and Han, 2006; Du et al., 2015). Kirdak et al. reported that when different doses of methylprednisolone were used topically to prevent the formation of peritoneal adhesions there was not any difference among the doses and that steroids did not have any effect in decreasing the formation of peritoneal adhesions (Durmus et al., 2007). In our study, during the surgical decompression of the median nerve the patients that were given methylprednisolone had statistically significant changes in their VAS and BSSS values as compared to patients who had undergone nerve decompression surgery only thereby supporting the literature.

Chitosan (CS) is one of the widely used naturally based polymers in wound healing applications. Carboxymethyl chitosan (CMCS) is a water-soluble CS derivative successfully used as a biomaterial in both research and clinical applications. Due to their favorable biocompatibility, no antigenicity, moisture retention, specific bioadhesion, and antibacterial ability, CS and CMCS are regarded as attractive materials for wound healing agents. (Wan et al., 2010; Liu et al., 2017) The CMCS derivatives can interact with cells which successfully result in cell growth/tissue regeneration and wound healing. They are also employed in the cosmetics production because of their moisture absorption-retention, antimicrobial and emulsion stabilizing characteristics. This work will highlight the most recent applications of $\mathrm{CMC}$ derivatives with antimicrobial, anticancer, antitumor, antioxidant and antifungal biological activities in various areas like wound healing, tissue engineering, and drug/enzyme delivery (Chen and Park, 2003)

In the study by Sun et al. when microscopic tissue examination was performed 15 days after the injury; wound healing was cleaner, more regular and had resulted in intensive reepithelialization layer in the group receiving carboxymethyl chitosan locally (Sun et al., 2014). In a study by Safak; hyaluronic acid (HA)/carboxymethyl cellulose (CMC), HA/sodium alginate $(\mathrm{NaAlg})$ and $\mathrm{HA} / \mathrm{CMC} / \mathrm{NaAlg}$ nanofiber surfaces generated with electric traction were used as surgical adhesion barriers and their performances were compared with other adhesion barriers with in vitro and in vivo studies. As a result, in vitro and in 
vivo studies proved that three nanofiber surfaces that were produced could be used as adhesion barriers. In our study, in the patient group where CMCS was used during surgery, both VAS and BSSS values were significantly different as compared with the patient group undergoing median nerve decompression only and these findings were supporting the literature. When the groups receiving CMCS and methylprednisolone during surgery were compared, a statistically significant difference could not be found.

In conclusion, when we compared the groups treated with CMCS and methyl prednisolone during surgical interventions for carpal tunnel syndrome with those who had undergone only surgery; we see that there was a significant decrease in the symptoms at three months. However, in order to follow-up adhesion related symptoms in the long run, we need more comprehensive prospective and histopathological studies.

\section{Conclusion}

In conclusion, when we compared the groups treated with CMCS and methyl prednisolone during surgical interventions for carpal tunnel syndrome with those who had undergone only surgery; we see that there was a significant decrease in the symptoms at three months. However, in order to follow-up adhesion related symptoms in the long run, we need more comprehensive prospective and histopathological studies.

Ethics committee approval: The Ordu University Clinical Research Ethics Committee approved the protocol of this study (KAEK 2018-210).

Peer-review: Externally peer-reviewed.

Author Contributions: Conception: T.Y. Design: T.Y. Supervision: T.Y. Fundings: T.Y.. Materials: T.Y.. Data Collection and/or Processing: T.Y. Analysis and/or Interpretation: T.Y. Literature Review: T.Y. Writing: T.Y. Critical Review : T.Y. Conflict of Interest: No potential conflict of interest relevant to this article was reported.

Financial Disclosure: The author declared that this study has received no financial support.

\section{References}

Alkan F, Koc Y, Celik I, Erol M, Aydin M.F. An Experimental Study Evaluating on the Efects of Flunixin Meglumlne and Mepiramine Maleate in the Prevenuon of Intraabdominal Adhesions in Rabbits. Vet Bil journal 2007;21:73-9.
Burns, J.W., Colt, M.J., Burgees, L.S., Skinner, K.C., Preclinical Evaluation of Seprafilm Bioresorbable Membrane, The European Journal of Surgery. Supplement $=$ Acta Chirurgica. Supplement 1996;577:40-8. i[s] :

Carlson H, Colbert A, Frydl J, Arnall E, Elliot M, Carlson N. Current options for nonsurgical management of carpal tunnel syndrome. Int J Clin Rheumtol 2010;5:129-42. is[ep?

Chen X, Park H. Chemical characteristics of Ocarboxymethyl chito- sans related to the preparation conditions. Carbohydr Polym 2003;53(4):355-59.

Du XH, Liu JQ, Xin K, Liu GH. Dexamethasone and sodium carboxymethyl cellulose prevent postoperative intraperitoneal adhesions in rats. Braz J Med Biol Res 2015;48(4):344-48.

Durmus AS, Han MC. Comparison of the effects of different concentrations of sodium carboxymethyl cellulose on prevention of intraabdominal adhesions in rats. Revue Med Vet 2006;157:53538.

Durmus AS, Yildiz H, Yaman M, Simsek H. The effects of heparin and pentoxifylline on prevention of intra- abdominal adhesions in rat uterine horn models: histopathological and biochemical evaluations. Revue Med Vet 2011;162:198-203.

Gelbermen R, Aranson D, Weisman M. Carpal Tunnel Syndrome. J. Bone and Joint Surg 1980;62:1181-84.

Gerritsen AA, de Krom MC, Struijs MA et al. Conservative treatment for carpal tunnel syndrome: a systematic review of randomized controlled trials. J Neurol 2002;249:272-80.

Hadler NM. Nerve entrapment syndromes. In: McCarty DJ, Kopman WJ (Editors). Arthritis and allied conditions. Lea and Febiger: Philadelphia 1993:P.1619-24.

Holmdahl, L., Eriksson, E., Eriksson, B.I., Risberg, B., Depression of Peritoneal Fibrinolysis During Operation is a Local Response to Trauma, Surgery 1998;123(5): 539-44.

Koc Y, Alkan F, Uyaroglu A. The use of sodium carboxymethylcellulose in the prevention of intraabdominal adhesions in rabbits. Turk J Vet Anim Sci 2003;27:965-70.

Liu Q, Huang Y, Lan Y, et al. Acceleration of skin regeneration in full- thickness burns by incorporation of bFGF-loaded alginate microspheres into a CMCS-PVA hydrogel. J Tissue Eng Regen Med 2017;11(5):1562-73. 
Milligan, D.W., Raftery, A.T. Observations on the Pathogenesis of Peritoneal Adhesions: A Light and Electron Microscopical Study, British Journal of Surgery $1974 ; 61(4): 274-80$.

Phalen GS. Reflections on 21 years experience with the carpal tunnel syndrome. JAMA; 1970. P. 2365.

Raftery, A.T., Effect of Peritoneal Trauma on Peritoneal Fibrinolytic Activity and Intraperitoneal Adhesion Formation, European Surgical Research, 1981;13(6). 397-401.

Rodgers, K.E., Dizerega, G.S., Function of Peritoneal Exudate Cells After Abdominal Surgery, Journal of Investigative Surgery 1993;6(1). 9-23.

Schnuriger, B., Barmparas, G., Branco, B.C., Lustenberger, T., Inaba, K., Demetriades, D., Prevention of Postoperative Peritoneal Adhesions: A Review of The Literature, The American Journal of Surgery 2011;201(1):111-21.

Scholten RJ, de Krom MC, Bertelsmann FW et al. Variation in the treatment of carpal tunnel syndrome. Muscle and Nerve 1997; 20: 1334-35.

Sun BK, Siprashvili Z, Khavari PA. Advances in skin grafting and treatment of cutaneous wounds. Science 2014;346(6212):941-45.

Ulvi H,Yiğiter R,Aygul R,Varoglu OA. Diagnosis and Treatment Approaches in Carpal Tunnel Syndrome. Van Medical Journal 2004;11(4):15560.

Wang G, Lu G, Ao Q, Gong Y, Zhang X. Preparation of cross-linked carboxymethyl chitosan for repairing sciatic nerve injury in rats. Biotechnol Lett 2010;32(1):59-66.

Yagcı I, Ucan H, Y1lmaz L, Yağmurlu F, Keskin D, Bodur H. Efficacy of Kinesiotaping on Symptoms, Hand Functions, and Hand Grip Strength in Carpal Tunnel Syndrome: A Single-Blind and Randomized Controlled Study. Turk J Phys Med Rehabil 2006;52:55-60. 